\title{
The effects of siRNA against RPL22 on ET-1-induced proliferation of human pulmonary arterial smooth muscle cells
}

\author{
KAI SUN ${ }^{1,2}$, HONG XUE ${ }^{1}$, HUI WANG ${ }^{1}$, QIANG WANG $^{1}$, XIANG-RONG ZUO ${ }^{1}$, \\ WEI-PING XIE ${ }^{1}$ and HONG WANG ${ }^{1}$ \\ Departments of ${ }^{1}$ Respiratory Medicine and ${ }^{2}$ Emergency Medicine, The First Affiliated \\ Hospital of Nanjing Medical University, Nanjing 210029, P.R. China
}

Received February 27, 2012; Accepted April 20, 2012

DOI: $10.3892 / \mathrm{ijmm} .2012 .992$

\begin{abstract}
The aim of this study was to investigate the effects of small interference RNA (siRNA) against ribosomal protein L22 (RPL22) on ET-1-induced proliferation of human pulmonary arterial smooth muscle cells (HPASMCs). HPASMCs were transfected with siRNA against RPL22, incubated in smooth muscle cell culture medium (SMCM) and ET-1. RPL22 gene expression and protein levels of HPASMCs were measured by real-time PCR and western blotting, respectively. PCNA, CCK-8 immunohistochemistry and flow cytometry analysis were used to evaluate HPASMC proliferation. Cyclin D1 (CCND1) expression was also assayed. Following transfection with siRNA against RPL22, RPL22 expression was significantly inhibited in the control and ET-1 groups. HPASMC proliferation was significantly suppressed by transfection with siRNA against RPL22. Moreover, CCND1 was also downregulated by inhibiting RPL22 expression. In conclusion, these data suggest that inhibition of RPL22 expression can suppress HPASMC proliferation and CCND1 expression. The effects of siRNA against RPL22 on HPASMC proliferation are considered to be mediated through inhibition of CCND1 expression.
\end{abstract}

\section{Introduction}

Pulmonary hypertension (PH) is an insidious and complex disease involving both the cardiovascular and pulmonary systems (1). It is a progressive disease characterized by progressive increasing of pulmonary vascular resistance, leading to right ventricular hypertrophy, right heart failure and fatal arrhythmia. According to the PH guideline of ESC/ERS 2009 and $\mathrm{ACCF} / \mathrm{AHA}$, pulmonary arterial hypertension $(\mathrm{PAH})$ is the most common series of $\mathrm{PH}(1,2)$. Although the imbalance

Correspondence to: Dr Hong Wang, Respiratory Medicine, The First Affiliated Hospital of Nanjing Medical University, Nanjing 210029, P.R. China

E-mail: hongwang_prof@sina.com

Key words: pulmonary arterial hypertension, pulmonary arterial smooth muscle cell, proliferation, ribosomal protein L22 between pulmonary artery vasoconstriction and vasodilatation is the pathophysiology base of PAH, the remodeling of pulmonary arteries represents a main pathological lesion associated with the disease. The abnormal remodeling of the pulmonary arterial wall includes intimal proliferative, medial hypertrophy, adventitial thickening and plexiform lesions, resulting in the obstruction of resistant pulmonary arteries (3).

The development of PAH involves a complex and heterogeneous constellation of multiple genetic, molecular, and humoral abnormalities, presenting a final manifestation of vascular remodeling in which fibroblasts, smooth muscle and endothelial cells all have a role (4). Studies have demonstrated that the proliferation of pulmonary arterial smooth muscle cells (PASMCs) are a significant feature of PAH (5). PASMC apoptosis is suppressed and proliferation is enhanced in experimental PAH (6). Recently, several groups have concluded that PAH may be viewed as a disease of excess proliferation and impaired apoptosis of PASMCs, similarly in some regards to neoplasia (7). Several mechanisms have been proposed for the involvement of PASMC abnormal proliferation in PAH, but the precise mechanisms are not clearly understood $(4,5,8)$.

The ribosome is a cellular organelle where mRNAs are translated into proteins in all eukaryotic cells. The ribosome has about 80 different proteins. Ribosomal proteins (RPs) are important constituents of the ribosome that have an essential role in the formation of a fully functional ribosome. It can be predicted that the defects in RPs can cause ribosome dysfunction (9). Although RPs are essential for cell growth, the effects of their mutations and their roles in human diseases have been ignored. With the advancing research during the past decade, more and more functions of RPs have been discovered. Studies have shown that RPL11 can form a complex binding with p53 to stabilize p53 protein. RPL11 expression can relieve HDM2mediated repression of p53 transactivation and activate endogenous p53 (10). The defects of RPs can lead to DiamondBlackfan anemia (DBA), an inherited red cell aplasia (11). RPL26 can bind p53 mRNA to augment its translation and bind Mdm2 to drive its degradation (12).

Ribosome protein L22 (RPL22), a component of the 60S eukaryotic ribosomal subunit, is one of the important proteins in the RP family. RPL22 deficiency may selectively upregulate $\mathrm{p} 53$ in $\alpha \beta$-lineage thymocytes by increasing p53 synthesis. Moreover, RPL22 deficiency has been reported to selectively 
arrest development of T cells at the $\beta$-selection checkpoint by inducing their death (13). Our preceding research showed that iptakalim, a selective $\mathrm{K}_{\text {АTP }}$ channel opener $(14,15)$, suppressed ET-1-induced HPASMC proliferation and significantly inhibited RPL22 expression in HPASMCs (16). It was presumed that RPL22 may be involved in the proliferation of HPASMCs. In the present study, siRNA against RPL22 was used to investigate the effects of RPL22 on HPASMC proliferation and the potential mechanisms.

\section{Materials and methods}

HPASMCs preparation and cultures. Approval for using human specimens in this study was obtained from the Jiangsu Province Public Hospital Ethical Review Board (Nanjing, China). Specimens of the human pulmonary arteries were obtained from the healthy lung segments of the patients undergoing pulmonary resection at the Department of Cardiothoracic Surgery, Jiangsu Province Hospital (Nanjing, China). Under sterile conditions, the intrapulmonary arteries (3rd-4th division) were dissected from adventitia and opened longitudinally. The endothelium was separated with a sterile scalpel blade, and the vessel was cut into $1-3 \mathrm{~mm}^{2}$ pieces. Pieces of the arteries were incubated in a culture bottle of SMCM with $20 \%$ FBS, $100 \mu \mathrm{g} / \mathrm{ml}$ streptomycin, $100 \mathrm{U} / \mathrm{ml}$ penicillin (all these were purchased from ScienCell Ltd.) at $37^{\circ} \mathrm{C}$ in an atmosphere of $5 \% \mathrm{CO}_{2}$ and $95 \%$ air. Cells were confluent after 4 weeks, then digested with $0.25 \%$ trypsin and subcultured. Cells from passages 4 and 6 were used for the subsequent experiments. The purity of HPASMCs in the primary cultures was confirmed by positive staining with a smooth muscle $\alpha$-actin antibody by immunocytochemistry as previously described (17).

The cells were randomly divided into six groups: control group, treated with culture medium alone; siRNA-RPL22 group, treated with siRNA-RP22; siNT group, treated with nontarget siRNA; ET-1 group, treated with $10 \mathrm{nM}$ ET-1, a suitable concentration for ET-1 to trigger HPASMC proliferation as previously described (17); siRNA-RPL22+ET-1 group, treated with siRNA-RPL22 plus 10 nM ET-1; siNT+ET-1 group, treated with nontarget siRNA plus $10 \mathrm{nM}$ ET-1. Before treatment, cells were cultured in serum-free medium for $24 \mathrm{~h}$ to be synchronized.

Small interference RNA (siRNA) transfection treatment. The RPL22 siRNA (ON-TARGETplus SMARTpool) and negative control siRNA (siCONTROL Non-Targeting siRNA pool) were purchased from Thermo Fisher Scientific, Inc. (Waltham, MA). One day prior to the experiment, cells were plated at $1 \times 10^{5}$ cells/well in 6-well plates at $2 \mathrm{ml}$ of medium overnight to achieve the desired density of $50-60 \%$ confluency. On the next day, the cells were transfected with siRNA (control siRNA and siRNA-RPL22, final concentration: $100 \mathrm{nM}$ ) using the X-tremeGENE siRNA transfection reagent (Roche, USA), according to the manufacturer's instructions: 500 pmol of siRNA and $10 \mu \mathrm{l} \mathrm{X}$-tremeGENE were diluted in $750 \mu \mathrm{l}$ of OptiMEM (Gibco-BRL Life Technologies) in one well. After pre-incubation for $25 \mathrm{~min}$ at $37^{\circ} \mathrm{C}$, both solutions were mixed and incubated for an additional $15 \mathrm{~min}$ at room temperature. The X-tremeGENE/siRNA mixture was subsequently overlaid onto the HPASMCs and incubated for $6 \mathrm{~h}$. Finally, $2 \mathrm{ml}$ of growth medium (10\% fetal bovine serum) per well replaced the mixture for further cultivation of the HPASMCs.

Real-time transcriptase-polymerase chain reaction (real-time $P C R)$. After treating cells as the above groups, transcripts were measured by TaqMan real-time quantitative reverse-transcriptase polymerase chain reaction (RT-PCR). Total-RNA was isolated from tissue samples using TriPure ${ }^{\circledR}$ Isolation Reagent (Roche, Indianapolis, IN, USA). The reverse transcription was performed in $300 \mathrm{ng}$ of total-RNA with TaqMan reverse transcription reagents kit (Takara, Japan). Real-time PCR was conducted as follows: $95^{\circ} \mathrm{C}$ at $10 \mathrm{~min}$ for 1 cycle, $95^{\circ} \mathrm{C}$ for $10 \mathrm{sec}$ and $60^{\circ} \mathrm{C}$ for $1 \mathrm{~min}$ for 40 cycles in the ABI PRISM ${ }^{\circledR}$ 7300 Real-time PCR system in a single capillary tube. Forward and reverse primers were each designed in a different exon of the target gene sequence, eliminating the possibility of amplifying genomic DNA. A positive result was determined by identifying the threshold cycle value at which reporter dye emission appeared above background. If the fluorescence signal was not detected within 40 cycles, the result was considered negative. The following gene primers were used: $\beta$-actin forward, 5'-TAAAGACCTCTATGCCAACACAGT-3' and reverse, 5'-CACGATGGAGGGGCCGGACTCATC-3'; RPL22 forward, 5'-CATGCCACTTAGGCCATGACT-3' and reverse, 5'-TGGTAGCCCCTTTCAGTTGTCTA-3'; cyclin D1 forward, 5'-GGTCTGCGAGGAACAGAAGTG-3' and reverse, 5'-TGCAGGCGGCTCTTTTTC-3'.

Western blot analysis. The HPASMCs treated as mentioned above after incubation were washed twice with ice-cold PBS and lysed in $200 \mu 1$ lysis buffer (Beyotime, China). The cell lysates were microcentrifuged at $14,000 \mathrm{rpm}$ for $5 \mathrm{~min}$ at $4^{\circ} \mathrm{C}$. After being heated for $5 \mathrm{~min}$ at $95^{\circ} \mathrm{C}, 40 \mu \mathrm{g}$ of denatured protein for each reaction was used to load a $12 \%$ polyacrylamide SDS-PAGE gel. Equal amounts of protein were transferred to PVDF membranes (Millipore, MA, USA). The membranes were blocked with $5 \%$ non-fat dry milk in Tris-buffered saline containing $0.1 \%$ Tween-20, and incubated with respective primary antibodies (RPL22 antibody 1:1,000 dilution, Abcam, USA; cyclin D1 antibody 1:1,000 dilution, Cell Signaling Technology, USA). The signals were developed using Super-Signal West Pico chemiluminescent substrate (Pierce, Rockford, IL, USA) and visualized by Quantity One 4.6.2 software. GAPDH was used as the control.

Cell counting kit-8. The HPASMCs as described above were planted into 96 -well plates in $200 \mu \mathrm{l}$ of cell culture medium at 3,000 cells/well supplemented with $2 \%$ FBS and incubated for 24 or $48 \mathrm{~h}$. Next, $10 \mu \mathrm{l}$ of CCK-8 reagent (Dojindo, Japan) was added to each well $2 \mathrm{~h}$ before the end of incubation. The optical density value (OD) of each sample was measured at a wavelength of $450 \mathrm{nM}$ on a microplate reader (Multiskan MK3, Thermo Lab Systems). The results of cell viability measurement were expressed as the absorbance at OD450.

Cell cycle phase determination using flow cytometry. HPASMCs were cultured in culture bottles and brought to quiescence as described above. After ET-1 (final concentrations $10^{-8} \mathrm{nM}$, Sigma) treatment for $24 \mathrm{~h}$, cells were trypsinized and centrifuged at $1,500 \mathrm{x} \mathrm{g}$ for $5 \mathrm{~min}$, and then were fixed 
A

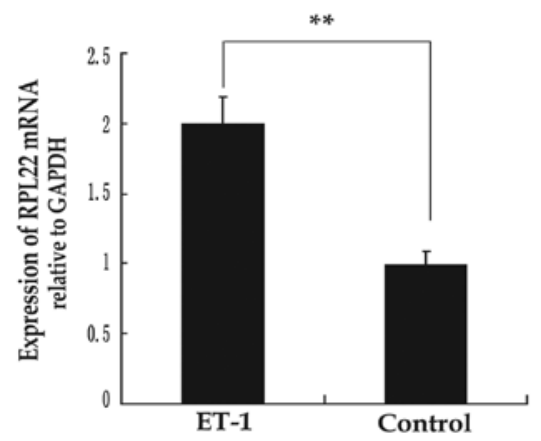

B

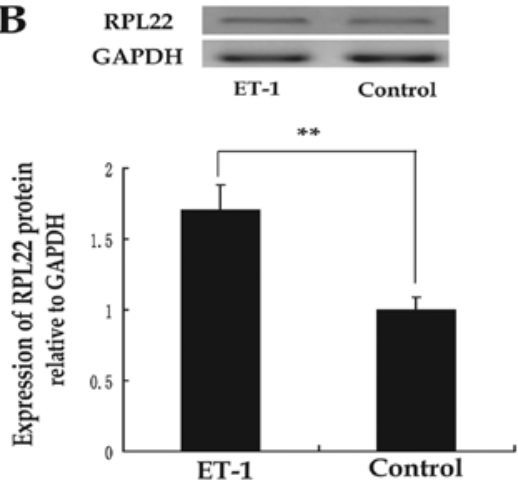

Figure 1. Expression of RPL22 induced by ET-1 in HPASMCs. Cultured HPASMCs were treated with ET-1 (10 ng/ml). (A) The expression of RPL22 mRNA level at $48 \mathrm{~h}$ after administration with ET-1 and medium alone evaluated by real-time PCR. (B) The expression of RPL22 protein at $30 \mathrm{~h}$ after administration with ET-1 $(10 \mathrm{ng} / \mathrm{ml})$ and medium alone was measured by western blot analysis. The graph shows the relative RPL22 protein levels normalized to GAPDH. Results are presented as the mean $\pm \mathrm{SD}$ of three independent experiments $(\mathrm{n}=3) .{ }^{* *} \mathrm{P}<0.01$.

A

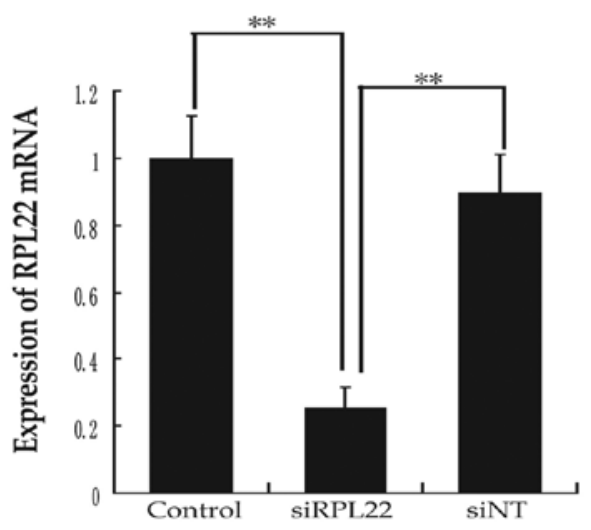

B RPL 22

GAPDH
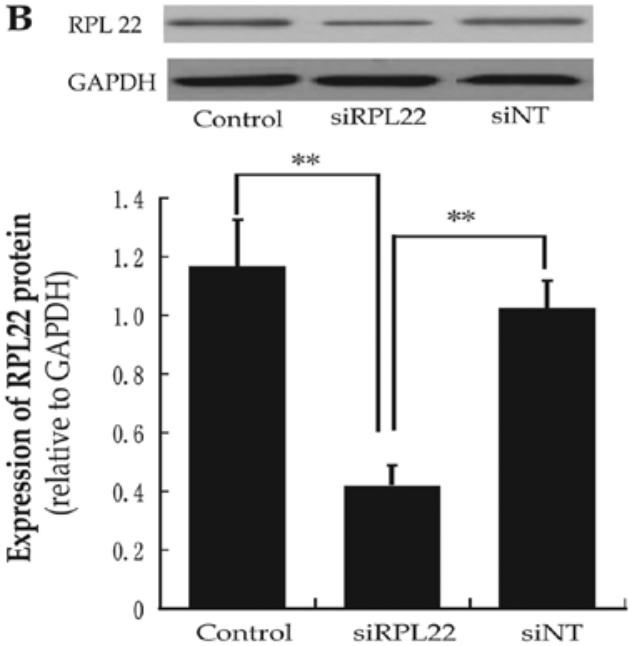

Figure 2. siRNA treatment reduced the expression of RPL22 in HPASMC. Cultured HPASMCs were treated with siRPL22 (RPL22 siRNA), siNT (negative siRNA) and control (medium alone) for the indicated times. (A) Relative RPL22 gene expression levels at $48 \mathrm{~h}$ after administration of different treatments detected by real-time PCR. (B) Expression of RPL22 protein measured by western blot analysis. The graph shows the relative RPL22 protein levels normalized to GAPDH. Results are presented as the mean \pm SD of three independent experiments $(n=3) .{ }^{* *} \mathrm{P}<0.01$.

with ice-cold $70 \%$ ethanol for $30 \mathrm{~min}$. Finally, the cells were stained with a mixture of propidium iodide (PI, $50 \mathrm{mg} / \mathrm{l}$ ) for $30 \mathrm{~min}$ at $4^{\circ} \mathrm{C}$ protected from light. The minimum of 105 events were collected and analyzed by flow cytometry in a FACScan (Becton-Dickinson, Heidelberg, Germany).

PCNA immunofluorescence assay. The HPASMCs were placed onto coverslips, which were covered in 24-well culture plates with polylysine. After treatment as described above and incubation for $72 \mathrm{~h}$, the HPASMCs were washed with PBS, fixed with $4 \%$ formaldehyde in PBS for $10 \mathrm{~min}$ and blocked in $1 \%$ BSA for $30 \mathrm{~min}$. The cells were incubated with an antibody against PCNA (1:100 dilution, Santa Cruz Biotechnology, Inc., Santa Cruz, CA, USA) overnight at $4^{\circ} \mathrm{C}$. Secondary antibodies used were coupled to goat anti-rabbit IgG Alexa Fluor 488 (1:800 dilution, A11008; Invitrogen, USA).

Statistical analysis. All data are presented as the mean \pm SD of three independent experiments. Differences between data sets were assessed by analysis of unpaired Student's t-test or
ANOVA and post-hoc tests (Student-Newman-Keuls) as indicated. Differences were considered to be significant at $\mathrm{P}<0.05$.

\section{Results}

Expression of RPL22 of HPASMCs with ET-1 condition. To determine RPL22 expression of HPASMC, real-time PCR and western blot analysis were used to assess RPL22 mRNA and protein levels of the cells cultured in SMCM alone or combined with ET-1. Results showed that RPL22 mRNA and protein were increased in ET-1 medium compared with control groups $(\mathrm{P}<0.05)$ (Fig. 1). Consequently, RPL22 expression increased in ET-1-treated HPASMCs.

Expression of RPL22 after siRNA interference treatment. To determine the effects of siRNA-RPL22 transfection on HPASMCs, RPL22 mRNA and protein levels were analyzed by real-time PCR and western blot analysis after cells were cultured for $48 \mathrm{~h}$. RPL22 mRNA levels were reduced to $25 \%$ in the siRNA-RPL22 group, compared with the siNT group. 


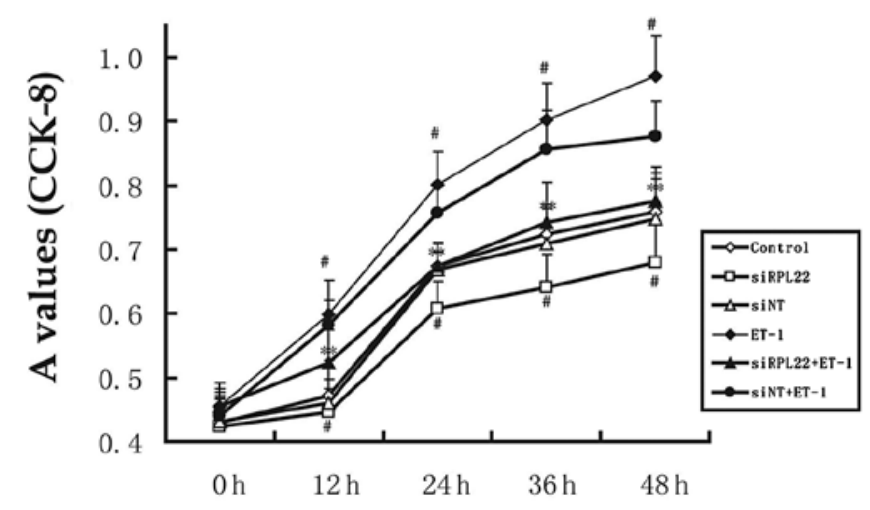

Figure 3. HPASMC proliferation assessed by the CCK- 8 assay. HPASMCs were treated as mentioned in Materials and methods. The proliferative capacity of HPASMCs in medium containing ET-1 was higher than in the control medium; while transient expression of RPL22 inhibited ET-1 induced proliferation of HPASMCs. After incubation for 24 and $48 \mathrm{~h}$, the effects in the siRPL22 group were significantly reduced compared with the controls $\left({ }^{* *} \mathrm{P}<0.05\right.$ vs. the control group; ${ }^{\#} \mathrm{P}<0.05$ vs. the ET-1 group).

RPL22 protein levels were reduced to $38 \%$ compared with the siNT group (Fig. 2). Consequently, RPL22 expression was efficiently inhibited by siRNA-RPL22 transfection.

Role of RPL22 on ET-1 induced HPASMCs proliferation and cell cycle alteration. To determine the effects of SiRNA-RPL22 on ET-1 induced proliferation of HPASMCs, the CCK-8 assay was used to assess HPASMC proliferation (Fig. 3). ET-1 significantly enhanced the proliferation of HPASMCs $(\mathrm{P}<0.05$ vs. control). However, transient expression of RPL22 inhibited ET-1-induced proliferation of HPASMCs. After incubation for 24 and $48 \mathrm{~h}$, the effects in the siRPL22 group were significantly lower compared with the controls $(\mathrm{P}<0.05)$.

Cell cycle analysis was performed to determine the cell cycle alteration in the HPASMCs treated as above. When treated with $10 \mathrm{nM}$ ET-1 alone, HPASMCs were propelled from the static phase (G0/G1) to the DNA synthesis $(\mathrm{S})$ and mitotic phase $(\mathrm{G} 2 / \mathrm{M})$. The ratios of the $\mathrm{S}$ and $\mathrm{G} 2 / \mathrm{M}$ phases were increased by 1.8 - and 1.5-fold, respectively compared to the control cells. However, the ratios of the $\mathrm{S}$ and G2/M phases were decreased insignificantly in the siRPL22 group cells compared to the control group (Fig. 4).

Role of RPL22 on ET-1-induced expression of PCNA. The expression of PCNA, a cell cycle-associated protein, was assessed by immunofluorescence (IF) analyses to further confirm the effects of siRNA-RPL22 on the proliferation of HPASMCs (Fig. 5). The percentages of PCNA protein positive cells were significantly reduced in the ET-1+siRPL22 group compared to the control and siNT groups. Consequently, siRNA-RPL22 inhibited HPASMC proliferation induced by ET-1.

Role of RPL22 expression of cyclin D1 in HPASMCs. Using real-time PCR and western blot analysis, the mRNA and protein expression of CCND1 were analyzed (Fig. 6). Statistically significant $(\mathrm{P}<0.05)$ increases compared to the control group were noted in the ET-1 group; statistically significant $(\mathrm{P}<0.05)$ changes compared to the siRPL22+ET-1 group were noted in the siRPL22 and siNT+ET-1 groups. Consequently, inhibition of RPL22 downregulated CCND1 expression.

\section{Discussion}

The present study provides the first direct evidence that RPL22 is involved in HPASMC proliferation. The development of PAH involves a complex constellation of multiple genes and factors, which interact with each other and subsequently activate intracellular signaling pathways that eventually result in the remodeling of pulmonary artery $(4,5,8)$. Many factors, such as ET-1 (18), serotonin (19), potassium channel (20), caveolins (21), VEGF (22), PDGF (23), bone morphogenetic protein (BMP) (24) involved in PAH progression are thought to play roles on HPASMC proliferation. However, the precise mechanisms of PAH are still not completely unclear. Pulmonary artery vasoconstriction and vascular remodeling are pathological bases of PAH. Pulmonary arterial smooth muscle hypertrophy, the main cause of pulmonary artery vascular remodeling, is characterized by enhanced HPASMC proliferation $(4,8)$.

Our previous study showed that iptakalim $(14,15)$, inhibited ET-1 synthesis in cultured endothelial cells and suppressed ET-1-induced proliferation of HPASMCs in vitro $(17,25)$. In a proteomic study, it was found that iptakalim significantly inhibited protein expression of RPL22 in HPASMCs (16). Therefore, we presumed that RPL22 may be involved in the proliferation of HPASMCs. In this study, we firstly demonstrated that HPASMC proliferation was suppressed by siRNA-RPL22 interference. To investigate the effects of RPL22 on HPASMC proliferation, ET-1 was used as an activation factor.

ET-1 is a vasoactive peptide upregulated in PAH patients and exerts diverse actions on HPASMCs by interacting with the $\mathrm{G}$ protein-coupled receptors $(26,27)$. It is synthesized and released particularly by endothelial cells and PASMCs in the pulmonary circle (28). Previous studies have confirmed that ET-1 acts as the most potent vasoconstrictor and directly modulates HPASMCs growth by acting as an autocrine and paracrine secretion mitogen (29). Its effects include cell proliferation and contraction and it mediates cellular effects via two receptors, $\mathrm{ET}_{\mathrm{A}}$ and $\mathrm{ET}_{\mathrm{B}}$. $\mathrm{ET}_{\mathrm{A}}$ receptor is the predominant subtype of HPASMCs. It acts as a strong growth-promoting factor affecting HPASMC proliferation. ET-1 has also been reported to have anti-apoptotic effects on HPASMCs (30). To assess the effects on HPASMCs, we chose ET-1 as the activation factor. In the present study, ET-1 significantly enhanced proliferation of HPASMCs.

The ribosome is a cellular organelle, where mRNAs carrying DNA message assemble with ribosomal subunits and associate with tRNA, responsible for protein synthesis. In all eukaryotic cells, the ribosome consists of a small 40S subunit and a large 60S subunit (31). These subunits are composed of 4 RNAs and $\sim 80$ distinct proteins (32). Ribosomal proteins are the basic structure of the ribosome. They play an essential role in the formation of a fully functional ribosome. RPL22 is a component of the 60S large subunit and is encoded by the RPL22 gene. It has been confirmed to be located on chromosome 1 in eukaryotes (9). In the present study, we designed an siRNA against the RPL22 gene to inhibit RPL22 expression. 

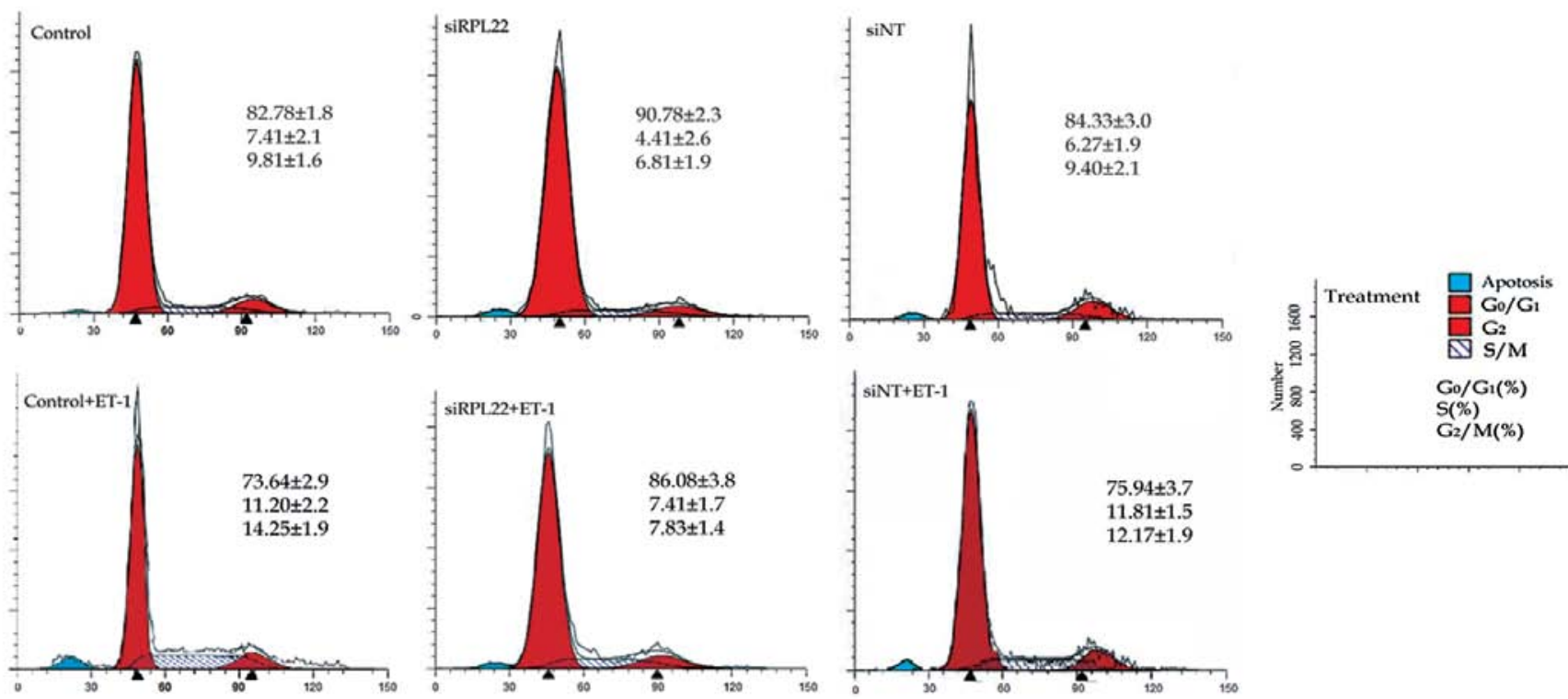

Figure 4. Role of siRNA-RPL22 transfection on cell cycle alterations of HPASMCs. HPASMCs were treated as mentioned above and the cell cycle of HPASMCs was analyzed by staining with propidium iodide and flow cytometry analyses. Representative graphs and the ratios of static phase (G0/G1), DNA synthesis $(\mathrm{S})$ and mitotic phase $(\mathrm{G} 2 / \mathrm{M})$. Data are presented as the mean $\pm \mathrm{SD}$ of three determinations in each treated group.
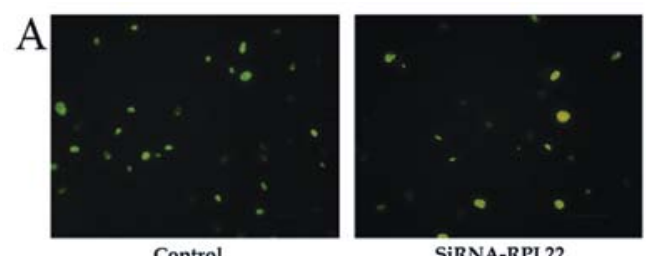

SiRNA-RPL22

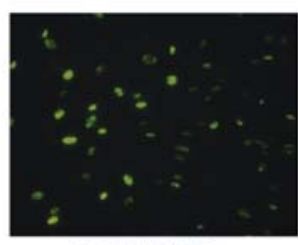

Control + ET-1

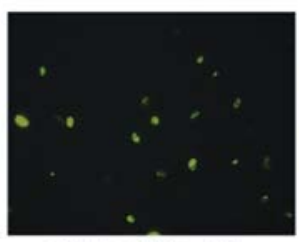

SiRNA-RPL22 + ET-1
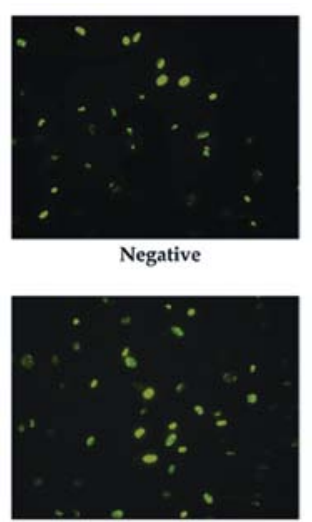

Negative + ET-1
B

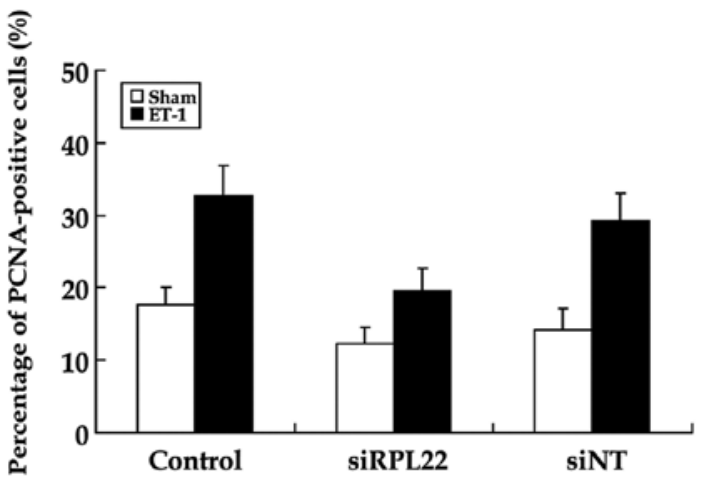

Figure 5. PCNA mRNA and protein expression of HPASMCs after treatment with ET-1, ET-1+siRPL22, siRPL22, ET-1+siNT, siNT and the control groups. (A) PCNA expression measured by immunofluorescence. The PCNA-positive cell percentages $48 \mathrm{~h}$ after administration of different treatments were measured by using an immunofluorescence assay. (B) The percentage of PCNA-positive HPASMCs was significantly reduced in the siRNA-RPL22 groups.

A

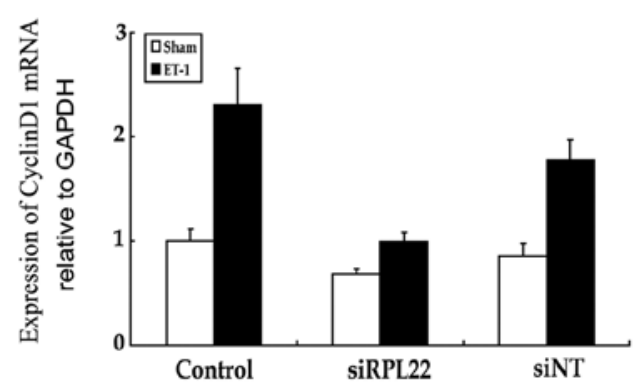

B
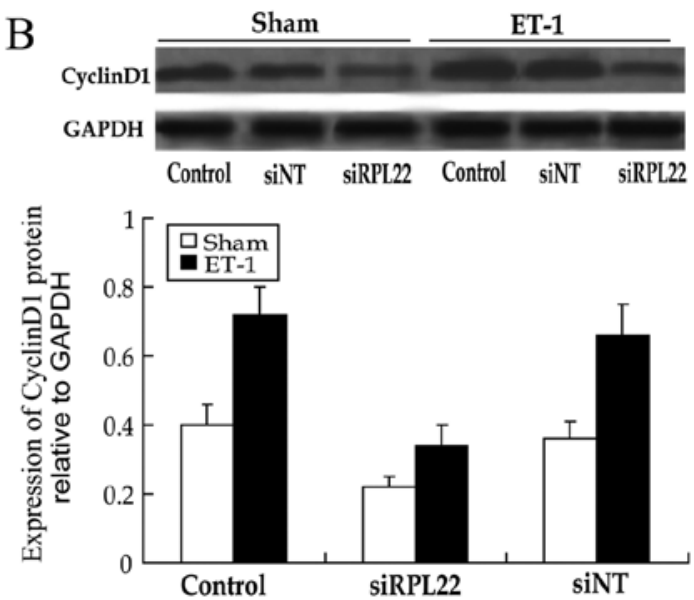

Figure 6. Cyclin D1 expression of HPASMCs evaluated by real-time PCR and western blot analysis. (A) CCND1 mRNA expression at $48 \mathrm{~h}$ after administration of different treatments evaluated by real-time PCR. (B) CCND1 protein expression at $48 \mathrm{~h}$ after administration of different treatments evaluated by western blot analysis. 
By real-time PCR and western blot analysis, results showed that siRNA-RPL22 transfection significantly inhibited RPL22 expression in HPASMCs. To confirm the effects of RPL22 deficiency on HPASMC proliferation, we examined HPASMC proliferation by different ways including PCNA immunocytochemistry, the CCK-8 test and flow cytometry analysis. Proliferating cell nuclear antigen (PCNA) is a protein that acts as a processivity factor for DNA polymerase. In this study, by PCNA immunocytochemical staining, we found that compared with the control group, HPASMC proliferation was significantly increased in the ET-1 group and was inhibited in the RPL22deficient groups with or without ET-1. The results of the CCK-8 assay and of the cell flow cytometry analysis also showed that HPASMC proliferation was significantly suppressed by inhibiting RPL22 expression. These results confirmed that inhibiting RPL22 expression can inhibit HPASMC proliferation and inhibit the proliferation effect of ET-1 on HPASMCs.

Furthermore, it was demonstrated that RPL22 deficiency can reduce CCND1 expression. CCND1 is one of the key proteins in the CCND protein family which control the progression of cells through the cell cycle by activating cyclin dependent kinases (CDKs) and plays an important role in the transition from the $\mathrm{G} 1$ to the $\mathrm{S}$ phase of the cell cycle during cell division. CCND1 is required in human cell proliferation and plays a key role at the G1 phase (33). In this study, we found that when HPASMCs were transfected with siRNARPL22, the CCND1 expression was significantly suppressed and the proliferation ability of HPASMCs was significantly impaired. This result show that RPL22 downregulation can inhibit CCND1 synthesis.

Ribosome gene disruption is not lethal as shown for other ribosomal proteins, and further supported by the fact that the effects of deficiency of some ribosomal protein can cause critical diseases (34). Our study showed that RPL22 deficiency can influence the synthesis of an proliferation factor of HPASMCs. RPL22 deficiency can inhibit CCND1 synthesis. It can be concluded that inhibiting CCND1 synthesis is one of the effects of RPL22 on HPASMC proliferation.

In summary, our present study confirms that RPL22 expression can be inhibited by siRNA-RPL22 transfection. The results demonstrate that siRNA-RPL22 can inhibit ET-1-induced HPASMC proliferation. These findings provide evidence to support that RPL22 is involved in HPASMC proliferation. This novel finding needs to be further explored.

\section{Acknowledgements}

We thank Dr G. Hu (Department of Pharmacology, Nanjing Medical University, Nanjing, China) for excellent technical assistance. This study was supported by the State Natural Science Funds Commission and by the Funds of the National Natural Science Foundation of China (Grant no. 30971319).

\section{References}

1. Galiè N, Hoeper MM, Humbert M, et al: Guidelines for the diagnosis and treatment of pulmonary hypertension. Eur Heart $\mathrm{J}$ 30: 2493-2537, 2009.

2. McLaughlin VV, Archer SL, Badesch DB, et al: ACCF/AHA 2009 expert consensus document on pulmonary hypertension. Circulation 119: 2250-2294, 2009.
3. Stewart S and Rassl D: Advances in the understanding and classification of pulmonary hypertension. Histopathology 54: 104-116, 2009.

4. Humbert M, Morrell NW, Archer SL, Stenmark KR, MacLean MR, Lang IM, Christman BW, Weir EK, Eickelberg O, Voelkel NF and Rabinovitch M: Cellular and molecular pathobiology of pulmonary arterial hypertension. J Am Coll Cardiol 43 (Suppl 12): S13-S24, 2004.

5. Mandegar M, Fung YC, Huang W, Remillard CV, Rubin LJ and Yuan JX: Cellular and molecular mechanisms of pulmonary vascular remodeling: role in the development of pulmonary hypertension. Microvasc Res 68: 75-103, 2004.

6. Gurbanov E and Shiliang X: The key role of apoptosis in the pathogenesis and treatment of pulmonary hypertension. Eur J Cardiothorac Surg 30: 499-507, 2006.

7. Archer SL, Weir EK and Wilkins MR: Basic science of pulmonary arterial hypertension for clinicians: new concepts and experimental therapies. Circulation 121: 2045-2066, 2010.

8. Morrell NW, Adnot S, Archer SL, et al: Cellular and molecular basis of pulmonary arterial hypertension. J Am Coll Cardiol 54 (Suppl 1): S20-S31, 2009.

9. Uechi T, Tanaka T and Kenmochi N: A complete map of the human ribosomal protein genes: assignment of 80 genes to the cytogenetic map and implications for human disorders. Genomics 72: 223-230, 2001.

10. Zhang Y, Wolf GW, Bhat K, Jin A, Allio T, Burkhart WA and Xiong Y: Ribosomal protein L11 negatively regulates oncoprotein MDM2 and mediates a p53-dependent ribosomal-stress checkpoint pathway. Mol Cell Biol 23: 8902-8912, 2003.

11. Gazda HT, Sheen MR, Vlachos A, Choesmel V, O'Donohue MF, Schneider H, Darras N, Hasman C, Sieff CA, Newburger PE, et al: Ribosomal protein L5 and L11 mutations are associated with cleft palate and abnormal thumbs in Diamond-Blackfan anemia patients. Am J Hum Genet 83: 769-780, 2008.

12. Ofir-Rosenfeld Y, Boggs K, Michael D, Kastan MB and Oren M: Mdm2 regulates $\mathrm{p} 53 \mathrm{mRNA}$ translation through inhibitory interactions with ribosomal protein L26. Mol Cell 32: 180-189, 2008.

13. Anderson SJ, Lauritsen JP, Hartman MG, Foushee AM, Lefebvre JM, Shinton SA, Gerhardt B, Hardy RR, Oravecz T and Wiest DL: Ablation of ribosomal protein L22 selectively impairs $\alpha \beta$ T cell development by activation of a p53-dependent checkpoint. Immunity 26: 759-772, 2007.

14. Pan Z, Huang J, Cui W, Long C, Zhang Y and Wang H: Targeting hypertension with a new adenosine triphosphate-sensitive potassium channel opener iptakalim. J Cardiovasc Pharmacol 56: 215-228, 2010.

15. Wang H, Tang Y and Zhang YL: Hypoxic pulmonary hypertension (HPH) and iptakalim, a novel ATP-sensitive potassium channel opener targeting smaller arteries in hypertension. Cardiovasc Drug Rev 23: 293-316, 2005.

16. Yang MX, Liu ZX, Zhang S, Jing Y, Zhang SJ, Xie WP, Ma L, Zhu CL and Wang H: Proteomic analysis of the effect of iptakalim on human pulmonary arterial smooth muscle cell proliferation. Acta Pharmacol Sin 30: 175-183, 2009.

17. Zhu YM, Zhang SJ, Xie WP, Li QL, Zhou YJ and Wang H: Iptakalim inhibited endothelin-1-induced proliferation of human pulmonary arterial smooth muscle cells through the activation of $\mathrm{K}_{\text {ATP }}$ channel. Vascul Pharmacol 48: 92-99, 2008.

18. Sakao S, Tatsumi K and Voelkel NF: Endothelial cells and pulmonary arterial hypertension: apoptosis, proliferation, interaction and transdifferentiation. Respir Res 10: 95-103, 2009.

19. Maclean MR and Dempsie Y: The serotonin hypothesis of pulmonary hypertension revisited. Adv Exp Med Biol 661: 309-322, 2010.

20. Burg ED, Remillard CV and Yuan JX: Potassium channels in the regulation of pulmonary artery smooth muscle cell proliferation and apoptosis: pharmacotherapeutic implications. Br J Pharmacol 153 (Suppl 1): S99-S111, 2008.

21. Zhao YY and Malik AB: A novel insight into the mechanism of pulmonary hypertension involving caveolin-1 deficiency and endothelial nitric oxide synthase activation. Trends Cardiovasc Med 19: 238-242, 2009.

22. Taraseviciene-Stewart L, Kasahara Y, Alger L, Hirth P, McMahon G, Waltenberger J, Voelkel NF and Tuder RM: Inhibition of the VEGF receptor 2 combined with chronic hypoxia causes cell deathdependent pulmonary endothelial cell proliferation and severe pulmonary hypertension. FASEB J 15: 427-438, 2001. 
23. Schermuly RT, Dony E, Ghofrani HA, Pullamsetti S, Savai R, Roth M, Sydykov A, Lai YJ, Weissmann N, Seeger W and Grimminger F: Reversal of experimental pulmonary hypertension by PDGF inhibition. J Clin Invest 115: 2811-2821, 2005

24. Lagna G, Nguyen PH, Ni W and Hata A: BMP-dependent activation of caspase- 9 and caspase- 8 mediates apoptosis in pulmonary artery smooth muscle cells. Am J Physiol Lung Cell Mol Physiol 291: L1059-L1067, 2006.

25. Xie WP, Wang H, Ding JH, Wang $\mathrm{H}$ and Hu G: Anti-proliferating effect of iptakalim, a novel $\mathrm{K}_{\text {ATP }}$ channel opener, in cultured rabbit pulmonary arterial smooth muscle cells. Eur J Pharmacol 511: 81-87, 2005 .

26. Rohit B, Rubin MT and Paul MH: Endothelial dysfunction in pulmonary hypertension. Circulation 109: 159-165, 2004.

27. Davie N, Haleen SJ, Upton PD, Polak JM, Yacoub MH, Morrell NW and Wharton $\mathrm{J}$ : $\mathrm{ET}_{\mathrm{A}}$ and $\mathrm{ET}_{\mathrm{B}}$ receptors modulate the proliferation of human pulmonary artery smooth muscle cells Am J Respir Crit Care Med 165: 398-405, 2002.

28. Tronic E and Webb DJ: Endothelium-derived endothelin-1. Pflug Arch Eur J Phy 459: 951-958, 2010.
29. Komuro I, Kurihara H, Sugiyama T, Yoshizumi M, Takaku F and Yazaki Y: Endothelin stimulates c-fos and c-myc expression and proliferation of vascular smooth muscle cells. FEBS Lett 238 : 249-252, 1988.

30. Jankov RP, Kantores C, Belcastro R, Yi M and Tanswell AK: Endothelin-1 inhibits apoptosis of pulmonary arterial smooth muscle in the neonatal rat. Pediatr Res 60: 245-251, 2006.

31. Robledo S, Idol RA, Crimmins DL, Ladenson JH, Mason PJ and Bessler M: The role of human ribosomal proteins in the maturation of rRNA and ribosome production. RNA 14: 1918-1929, 2008.

32. Hernandez-Verdun D: Assembly and disassembly of the nucleolus during the cell cycle. Nucleus 2: 189-194, 2011.

33. Stacey DW: Cyclin D1 serves as a cell cycle regulatory switch in actively proliferating cells. Curr Opin Cell Biol 15: 158-163, 2003.

34. McGowan KA, Li JZ, Park CY, Beaudry V, Tabor HK, Sabnis AJ, Zhang W, Fuchs H, de Angelis MH, Myers RM, et al: Ribosomal mutations cause p53-mediated dark skin and pleiotropic effects. Nat Genet 40: 963-970, 2008 